\title{
Analytical expression for the quantum dot contribution to the quasistatic capacitance for conduction band characterization
}

\author{
Ma Buda, ${ }^{1, a)}$ G. Iordache, ${ }^{1}$ S. Mokkapati, ${ }^{2}$ Lan Fu, ${ }^{2}$ G. Jolley, ${ }^{2}$ H. H. Tan, ${ }^{2}$ C Jagadish, ${ }^{2}$ \\ and $\mathrm{Mi} \mathrm{Buda}^{3}$ \\ ${ }^{1}$ National Institute of Material Physics, Magurele, P.O. Box MG7, Romania \\ ${ }^{2}$ Research School of Physical Sciences and Engineering, The Australian National University, \\ Canberra, ACT 0200, Australia \\ ${ }^{3}$ Faculty of Applied Chemistry and Materials Science, "Politehnica" University of Bucharest, \\ Calea Grivitei 132, 010737, Romania
}

(Received 29 December 2007; accepted 26 May 2008; published online 30 July 2008)

\begin{abstract}
This paper demonstrates an analytical expression for the quasistatic capacitance of a quantum dot layer embedded in a junction, where the reverse bias is used to discharge the initially occupied energy levels. This analysis can be used to determine the position and the Gaussian homogeneous broadening of the energy levels in the conduction band, and is applied for an InGaAs/GaAs quantum dot structure grown by metal organic chemical vapor deposition. It is shown that the Gaussian broadening of the conduction band levels is significantly larger than the broadening of the interband photoluminescence (PL) transitions involving both conduction and hole states. The analysis also reveals a contribution from the wetting layer both in PL and modeled $C$ - $V$ profiles which is much stronger than in typical molecular beam epitaxy grown dots. The presence of a built-in local field oriented from the apex of the dot toward its base, contrary to the direction expected for a strained dot with uniform composition (negative dipole), is also derived from fitting of the $C$ - $V$ experimental data. (C) 2008 American Institute of Physics. [DOI: 10.1063/1.2959681]
\end{abstract}

\section{INTRODUCTION}

The special features arising from quantization effects in zero-dimensional nanostructures are of great interest for a large variety of applications in electronics and integrated optoelectronics: memory devices, laser diodes, infrared detectors, etc. Consequently, the energy level characterization is a key issue for device design and for the physical understanding of the processes specific to the quantum nature of the dots. Theoretical calculations are cumbersome and their results depend considerably on many input parameters that are not well known, such as band discontinuities at the heterojunction between the strained dot material and the surrounding matrix, dot shape, and composition. One of the most suited methods for extracting experimentally the band structure is the quasistatic capacitance spectroscopy. ${ }^{1-8}$ It involves fitting the measured $C-V$ experimental plots for a structure, where the initially occupied quantized levels can be discharged by applying a reverse bias. It has also been widely applied for the determination of band offsets in quantum well (QW) heterostructures. ${ }^{9-11}$ While the capacitance spectroscopy technique provides information about the band structure (quantized levels and band discontinuities), the admittance spectroscopy brings an additional insight into the dynamics of the carrier transport through the structure. ${ }^{2,3,12-16}$

The energy band structure can then be compared with the results from theoretical models, providing a very useful tool for their validation and development. This paper is focused on the derivation of an analytical expression for the

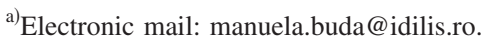

quantum dot (QD) contribution to the quasistatic capacitance and its use for characterizing the conduction band structure for a particular self-assembled QD system grown by metal organic chemical vapor deposition (MOCVD). As the analysis of the electronic band structure in III-V semiconductor quantum confined structures is much easier than for holes, the logical approach is to use QD layers embedded in a $n$-type structure for electronic level characterization using capacitance spectroscopy $(C-V)$ and photoluminescence (PL) for extracting the hole band structure. Capacitance spectroscopy probes only one band, while PL arises from transitions involving both electronic and heavy or light hole bands.

\section{EXPERIMENTAL}

$\mathrm{In}_{0.50} \mathrm{Ga}_{0.50} \mathrm{As} / \mathrm{GaAs} \mathrm{QDs}$ are grown in the StranskiKrastanow mode by MOCVD. ${ }^{17}$ Two $p^{+}-n$ structures were epitaxially grown on GaAs under similar conditions. The first structure acts as a reference, while in the second structure a single QD self-assembled layer is introduced in the middle of the $0.4 \mu \mathrm{m}$ thick $n$-GaAs matrix layer. The structure is designed such that for $0 \mathrm{~V}$ applied bias the QD layer lies outside the depleted region and when the reverse bias is increased, the charge from the QD system is removed and the depletion layer moves further into the GaAs matrix material. The details of the layer structure are given in Fig. 1. The PL measurements are performed at room temperature and $77 \mathrm{~K}$ using a green $\mathrm{He}-\mathrm{Ne}$ laser $(543.5 \mathrm{~nm})$ and an InGaAs photodiode after etching away the top $p^{++} \mathrm{GaAs}$ layer because it absorbs the PL light emitted from the QD region. The temperature dependent $C-V$ measurements were performed using a closed cycle (Ebara) He cooled Janis cry- 


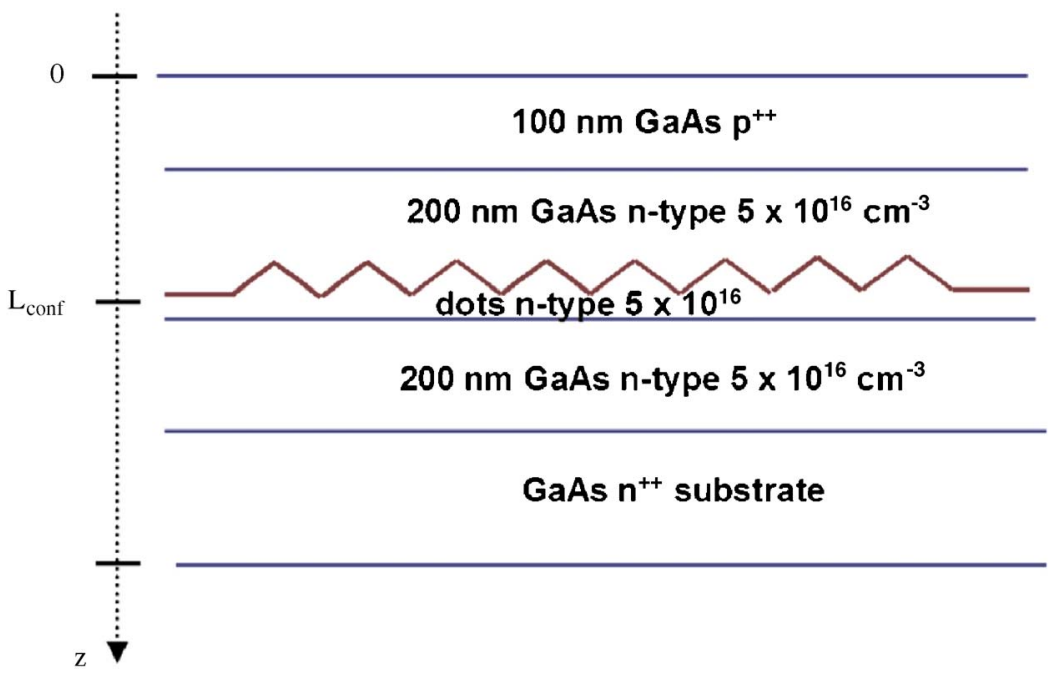

FIG. 1. (Color online) Layer structure used for capacitance spectroscopy characterization. A similar reference structure is grown separately without the QD layer. ostat system at $100 \mathrm{~Hz}, 1 \mathrm{kHz}, 10 \mathrm{kHz}$, and $100 \mathrm{kHz}$ using an Agilent capacitance bridge. The contact area is circular with a diameter of $780 \mu \mathrm{m}$.

For band structure characterization, the $C-V$ measurements must be made under quasistatic conditions, i.e., for frequencies smaller than the critical frequency for which the carrier population of the quantized levels can follow the changes in the small signal that is applied for capacitance measurement over the slowly varying reverse bias. The capacitance spectroscopy technique is a simplified version of the admittance spectroscopy method that involves measuring the real and imaginary parts of the admittance (capacitance and conductance) as functions of frequency, temperature, and applied bias. In $C$ - $V$ measurements, for quasistatic conditions (low frequency) the QD contribution to the capacitance no longer depends on the frequency and the corresponding conductance is zero. At high frequencies, the QD energy level population cannot follow the small signal variations of the bias applied for admittance measurement and the QD contribution to both capacitance and conductance is zero. The transition from high frequency to quasistatic conditions is marked by a step in the capacitance versus frequency plot, the characteristic frequency being determined by the response time of the quantum confined system for a particular reverse bias and temperature. The step in capacitance is accompanied by a peak in conductance, the two being connected by the Kramers-Kronig relationships.

\section{EXPERIMENTAL RESULTS}

Figure 2 shows the PL spectra for both the reference and the QD structures at 300 and $77 \mathrm{~K}$, respectively. The PL peaks of the reference structure show a GaAs related peak at $872 \mathrm{~nm}(1.42 \mathrm{eV})$ at RT and $827 \mathrm{~nm}(1.5 \mathrm{eV})$ at $77 \mathrm{~K}$, respectively. These peaks are also present in the QD structure but with a much smaller relative intensity_due to the efficiency of the PL processes in the wetting layer and in the QDs themselves. At longer wavelengths, broad defect related PL peaks due to the GaAs epilayer and substrate are also present in the reference structure with much lower intensity. These peaks are also absent in the QD structure. The intensity of the $1 e-1 h h$ wetting layer emissions at $983 \mathrm{~nm}(1.26$
$\mathrm{eV})$ for RT and at $947 \mathrm{~nm}(1.31 \mathrm{eV})$ at $77 K$ is unusually strong relatively to the QD PL and its strength increases significantly at lower temperatures for the same value of the excitation density. Even if the density of states in the wetting layer is much larger than that for the QD region, its population is considerably decreased due to the fact that the wetting layer states are situated higher in energy with respect to the common quasi-Fermi level. Especially at low temperatures, these energy barriers should be more effective and should reduce the wetting layer contribution in PL if the assumption of a common quasi-Fermi level is still valid. Instead, the wetting layer contribution is considerably stronger at lower temperatures. The QD PL at RT is deconvoluted using Gaussian dependencies to account for size fluctuations according to the following expression: $\mathrm{PL}=C_{o}$ $+\sum_{1}^{3} C_{i} e^{-\left(x-x_{o i}\right)^{2} / 2 w_{i}^{2}}$ and the extracted parameters from the corresponding interband transitions are as follows: $E_{1}$ $=1.054 \mathrm{eV}, w_{1}=32 \mathrm{meV}, E_{2}=1.130 \mathrm{eV}, w_{2}=56 \mathrm{meV}, E_{3}$ $=1.263 \mathrm{eV}$, and $w_{3}=35 \mathrm{meV}$. The first two transitions are related to the QD region, while the last transition corresponds to the $1 e-1 h h$ wetting layer peak. The size fluctuations are reflected in the width of the corresponding PL peaks through the $w$ parameters. Possibly, the $E_{2}$ excited state transition is a superposition of two different transitions that cannot be resolved due to size fluctuations.

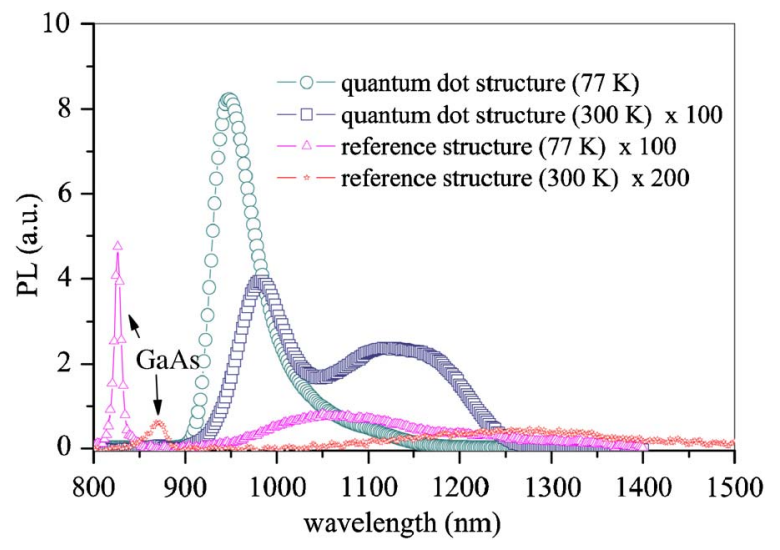

FIG. 2. (Color online) PL spectra for the reference and QD structures at room temperature and $77 \mathrm{~K}$, respectively. 


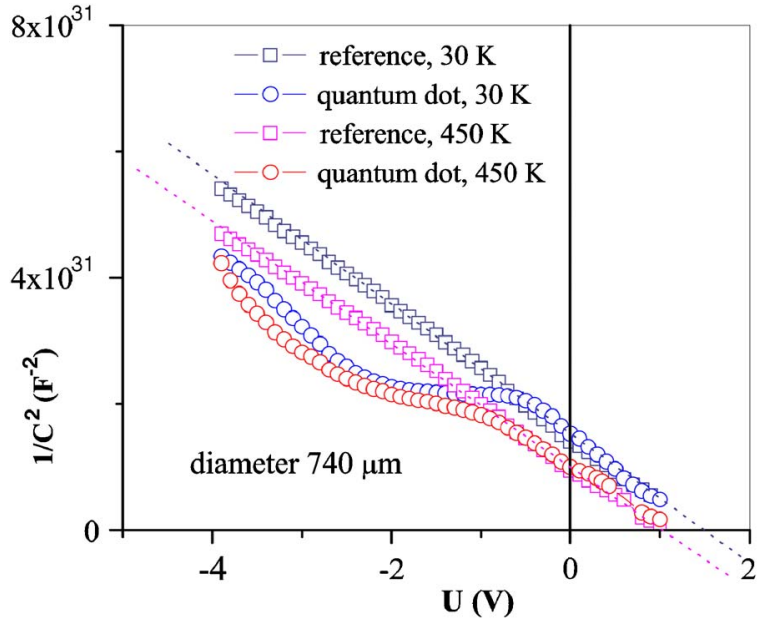

FIG. 3. (Color online) $1 / C^{2}-V$ plots at 450 and at $30 \mathrm{~K}$, respectively.

Figure 3 shows the plots of $1 / C^{2}$ versus the applied voltage at 45 and $30 \mathrm{~K}$ for the reference QD structures, respectively. From the slope of the plots for the reference structures, we extract a doping concentration of $5.8 \times 10^{16} \mathrm{~cm}^{-3}$ in the $n$-type GaAs epilayer. The same slope can be extracted from the QD structures but for a smaller range of voltages before the space charge region reaches the layer with energy confinement. The interception with the voltage axes is $1.47 \mathrm{~V}$ for both the reference and QD structures at $30 \mathrm{~K}$ and $1.08 \mathrm{~V}$ at $450 \mathrm{~K}$, respectively, and gives the built-in junction voltage $V_{b}$ due to the difference in doping between the $p^{++}$top GaAs contact layer and $n$-type $0.4 \mu \mathrm{m}$ thick GaAs epilayer.

Figures 4(a) and 4(b) show the experimental and modeled $C-V$ plots for the QD structure at 30 and $450 \mathrm{~K}$, respectively. The step profiles show the characteristic constant value of the capacitance of $220 \mathrm{pF}$, corresponding to the position of the space charge region inside the layer with energy confinement. The capacitance stays constant until the existing charge in the wetting layer and QDs is emptied and then drops as the space charge region extends further away from the position of the QD layer $L_{\text {conf }}$ (see Fig. 1), which is $0.2 \mu \mathrm{m}$ for our particular structure. As will be shown in Sec. $\mathrm{IV}$, the constant value of the capacitance of $220 \mathrm{pF}$ is mainly determined by the geometric capacitance $C=\varepsilon S / L_{\text {conf }}$ and does not depend on temperature.

The values for the energy confinements obtained from the modeling of the $C-V$ plots shown in Fig. 5 are $E_{\text {dot } 1}$ $=0.180 \mathrm{eV}, \sigma_{1}=180 \mathrm{meV}, E_{\mathrm{dot} 2}=0.375 \mathrm{eV}, \sigma_{2}=200 \mathrm{meV}$, and $E_{w l}=0.090 \mathrm{eV}$. Figures 5(a) and 5(b) show the potential and field profiles for different values of the applied bias (a) $V=+0.43 \mathrm{~V}$ and (b) $V=-0.20 \mathrm{~V}$, respectively. They will be discussed in Sec. IV.

\section{MODEL AND DISCUSSION}

We used a model similar to the one developed in literature initially for the determination of band offsets for QW heterostructures, ${ }^{9-11,18}$ which we extended for QD structures $^{3,5,7}$ by deriving an analytical expression for the contribution of the QDs to the quasistatic capacitance. This expression is very useful for the physical understanding of the phenomena and thus for weighing the contribution of the
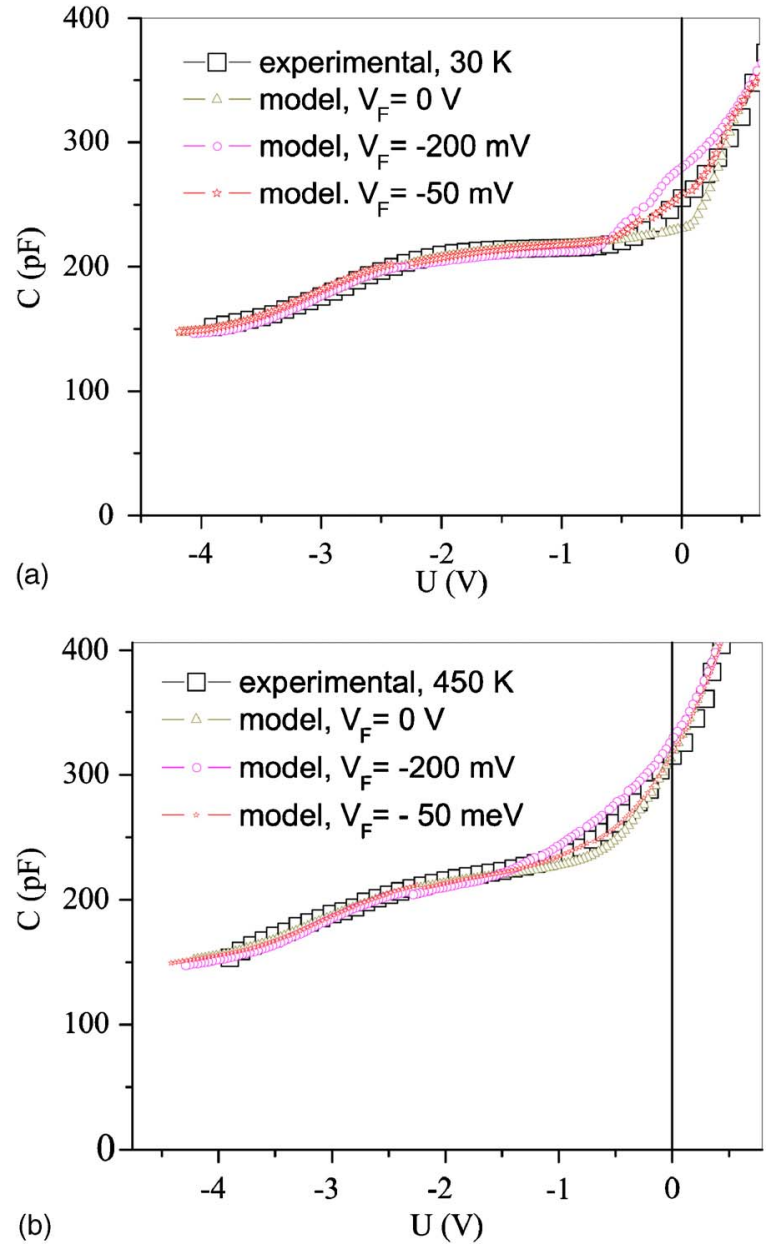

FIG. 4. (Color online) Experimental and modeled $C-V$ plots for the QD structure at (a) 30 and (b) $450 \mathrm{~K}$, respectively.

dot layer relative to the wetting layer capacitance. Unlike previous observations, in our samples the wetting layer contribution is important both in PL and capacitance spectra and must be accounted for. We also included the contribution of a local field for a better fit of the experimental plot for the range of data where the step in the $C$ - $V$ profile first appears, i.e., when the space charge region first encounters the QD layer and the discharging of its level begins.

\section{A. Exact solution of the Poisson equation for a "delta"-type thin layer with energy confinement}

We start with the one-dimensional Poisson equation for a $n$-doped material,

$$
\frac{d}{d z}\left\{\left[\varepsilon_{o} \varepsilon(z) \frac{d}{d z}\right] \Phi(z)\right\}=-q\left[N_{D}^{+}(z)-n(z)\right],
$$

$$
N_{D}^{+}(z)=\frac{N_{D}}{1+g_{n} e^{\left(E_{F}-E_{d}\right) / E_{t}}},
$$

where $\Phi$ is the electric potential, $\varepsilon$ and $\varepsilon_{0}$ are the absolute and relative permittivities, respectively, $N_{D}^{+}$is the concentration of ionized donors in the bulk material, $q$ is the elementary charge, and $n(z)$ is the $z$-dependent carrier concentration. The boundary conditions are $\Phi=V+V_{b}$ at $z=0$ and $\Phi=0$ at 

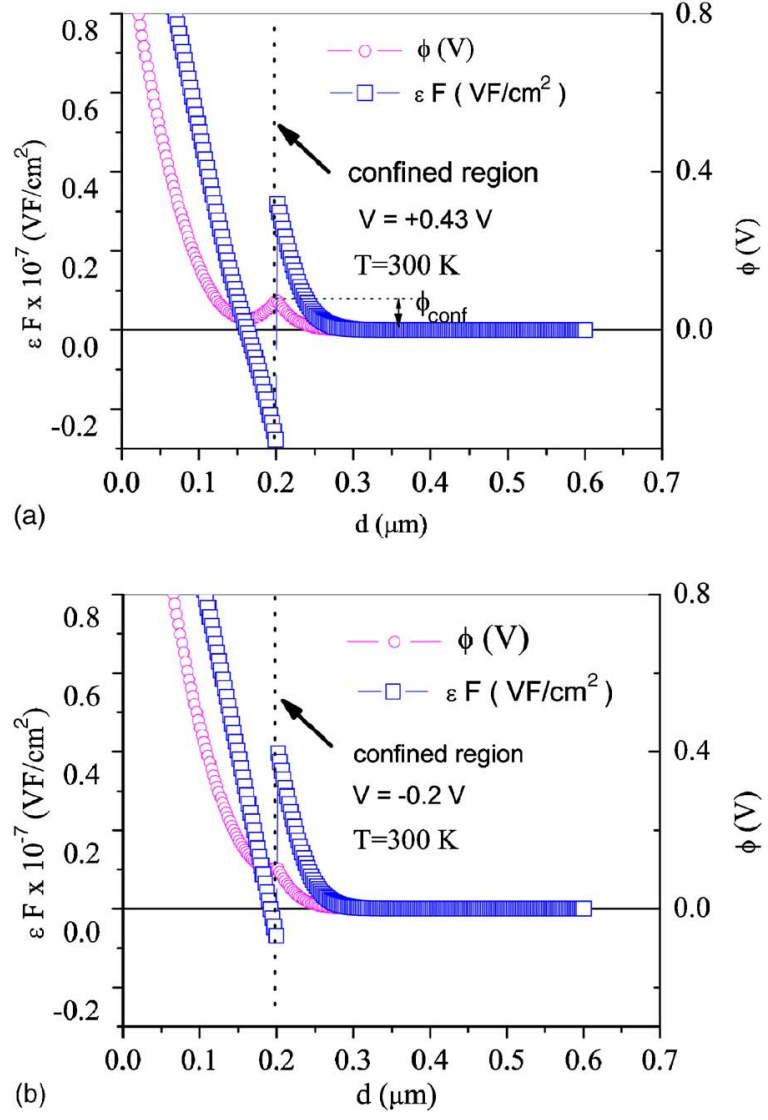

FIG. 5. (Color online) Potential and field profiles for different values of the applied bias (a) $V=+0.43 \mathrm{~V}$ and (b) $V=-0.20 \mathrm{~V}$, respectively.

$z=\infty$, where $V$ is the externally applied bias and $V_{b}$ is the built-in voltage of the junction.

For the $n$-type bulk GaAs epilayer, the three-dimensional carrier concentration is given by

$$
\begin{aligned}
& n(z)=n_{b}(z)=\frac{2}{\sqrt{\pi}} N_{c} \int_{0}^{\infty} \frac{\sqrt{\eta}}{e^{\eta-\eta_{F}}} d \eta \approx N_{c} e^{\left(E_{F}-E_{d}\right) / E_{t}}, \\
& N_{c}=2\left(\frac{m k T}{2 \pi \hbar^{2}}\right)^{3 / 2}\left(\mathrm{~cm}^{-3}\right),
\end{aligned}
$$

where $m$ is the electron effective mass, $k$ is the Boltzmann constant, and $T$ stands for temperature. $E_{F}$ is the common Fermi level for the whole structure corresponding to a given applied bias $V$. For the thin layer with energy confinement at $z=L_{\text {conf }}$, the delta-type sheet carrier concentration $n_{\text {conf }}$ is given by the sum of the wetting layer and QD contributions,

$$
\begin{aligned}
n_{\text {conf }}= & \frac{m_{c} k T}{\pi \hbar^{2}} \ln \left[1+e^{\left(E_{F}-\Phi_{\text {conf }}+E_{1}\right) / E_{t}}\right]+2 N_{\mathrm{dot}} \\
& \times \sum_{i=1}^{N \text { levels }} g_{i}\left[\frac{1}{\sqrt{2 \pi} \sigma_{i}} \int_{-\infty}^{\infty} e^{-\left(E-\Phi_{\text {conf }}+E_{\mathrm{dot} i}\right)^{2} / 2 \sigma_{i}^{2}}\right. \\
& \left.\times \frac{1}{\left[1+e^{\left(E-E_{F}\right) / E_{t}}\right]} d E\right]\left(\mathrm{cm}^{-2}\right),
\end{aligned}
$$

where $E_{1}$ is the confinement energy of the wetting layer single energy level and $E_{\mathrm{dot} i}$ are the confinement energies corresponding to the quantized dot energy levels relative to the bulk conduction band edge. $E_{t}$ is the thermal energy $k T / q$, where $q$ is the elementary charge.

The first term in Eq. (3) accounts for the contribution of the wetting layer which has a single energy level in the conduction band. The second term describes the contribution of $N_{\text {levels }}$ QD levels that are broadened due to Gaussian size fluctuations characterized by the $\sigma_{i}(\mathrm{meV})$ parameter for each level.

Equation (1) is solved using the bvp4c MATLAB solver for two point boundary ordinary differential equations for each value of the applied bias $V$. First, we write the Poisson equation as a system of two first order differential equations involving the potential and the electric field as

$$
\begin{aligned}
& y_{1}(z)=-\Phi(z), \\
& y_{2}(z)=-\varepsilon_{0} \varepsilon(z) \frac{d \Phi}{d z},
\end{aligned}
$$

and after solving it we extract the charge $Q$ in the structure for each applied bias $V$ as: $Q(V)=y_{2}(0)-y_{2}(\infty)$ together with the electric potential and field profile in the structure. The quasistatic capacitance is then obtained by the numerical derivation of the $Q(V)$ dependency. Figure 5 shows typical potential and field profiles using this model for two values of the applied bias: first, $V=+0.43 \mathrm{~V}$ [Fig. 5(a)] when the QD region lies outside the space charge region and second [Fig. $5(b)]$ for $V=-0.2 \mathrm{~V}$ when the space charge region extends over the QD thin layer. The other fitting parameters are those used for fitting the $C$ - $V$ plots in Figs. 4(a) and 4(b).

\section{B. Analytical solution for a delta-type thin layer embedded in a uniform region with doping $N_{D}$}

Without the contribution of the delta-type layer the thickness of the space charge region $W$ is given by $W$ $=\sqrt{2 \varepsilon\left(V+V_{b}\right) / q N_{D}}$. This is true for the reference structure for all values of the applied bias and for the QD structure when the applied bias is small, such that the thickness of the space charge region does not reach the QD layer. We now derive the expressions for the quasistatic capacitance of the structure with energy confinement at $z=L_{\text {conf }}$ for two limit cases:

\section{The delta-type layer with energy confinement is located inside the space charge region $W>L_{\text {conf }}$}

Using Eqs. (A4), (A7), and (A11) in the Appendix (see Ref. 18) that relate the real and imaginary parts of the admittance of the structure to the carrier population of the quantized levels and considering the Fermi-Dirac occupation probability $f_{0}$ for the wetting layer-i.e., a $\mathrm{QW}$ with a single level with the density of states $D_{1}=m / \pi \hbar^{2}$ and

$$
n_{\mathrm{QW}}=\int_{E_{1}}^{\infty} D_{1} f_{01} d E=\frac{k T m_{c}}{\pi \hbar^{2}} \ln \left[1+e^{\left(E_{F}-\Phi_{\text {conf }}+E_{1}\right) / E_{t}}\right],
$$

following again Ref. 18 for quasistatic conditions $(w \rightarrow 0)$, the analytical expression for the admittance of a QW thin layer is obtained as 


$$
\lim _{\omega \rightarrow 0} C_{\mathrm{QW}}=\frac{\varepsilon S\left[1+\frac{q^{2} m_{c}}{\varepsilon \pi \hbar^{2}}\left(W-L_{\mathrm{conf}}\right) \sum_{i} f_{0}\left(E_{\mathrm{wl} i}\right)\right]}{W\left[1+\frac{L_{\mathrm{conf}}}{W} \frac{q^{2} m_{c}}{\varepsilon \pi \hbar^{2}}\left(W-L_{\mathrm{conf}}\right) \sum_{i} f_{0}\left(E_{\mathrm{wl}} i\right)\right]},
$$

$$
\lim _{\omega \rightarrow 0} G_{\mathrm{QW}}=0,
$$

where we define $E_{\mathrm{wl} i}=\Phi_{\text {conf }}-E_{i}$ for the wetting layer and $E_{d i}$ as $\Phi_{\text {conf }}-E_{\text {dot } i}$ for the QDs, respectively, as shown in Fig. 6.

It remains now to evaluate the thickness of the space charge region $W$ when it includes a delta-type region with energy confinement. It is obtained by solving the transcendental Eq. (8) where $n_{\text {conf }}=n_{\mathrm{QW}}+n_{\text {conf dots }}$ depends on $W$ through the potential $\Phi_{\text {conf }}$ at the position of the QD layer $L_{\text {conf }}$,

$$
\begin{aligned}
& \Phi_{\text {conf }}=q \frac{N_{D}}{2 \varepsilon}\left(W-L_{\text {conf }}\right)^{2}, \\
& -(V+V)_{b}=q \frac{N_{D} W^{2}}{2 \varepsilon}-q \frac{L_{\text {conf }} n_{\mathrm{conf}}}{\varepsilon} .
\end{aligned}
$$

For the QD layer, the carrier density is given by

$$
\begin{aligned}
n_{\text {conf dots }}= & 2 N_{\mathrm{dot}} \times \sum_{i=1}^{N \text { levels }} g_{i}\left[\frac{1}{\sqrt{2 \pi} \sigma_{i}} \int_{-\infty}^{\infty} e^{-\left(E-\phi_{\mathrm{conf}}+E_{\mathrm{dot}}\right)^{2} / 2 \sigma_{i}^{2}}\right. \\
& \left.\times \frac{1}{\left(1+e^{\left.\left(E-E_{F}\right) / E_{t}\right)}\right.} d E\right]\left(\mathrm{cm}^{-2}\right)
\end{aligned}
$$

where $N_{\text {dot }}$ is the areal dot density which was estimated from atomic force microscopy studies on uncapped samples to be $\sim 3 \times 10^{10} \mathrm{~cm}^{-2}$ and $g_{i}$ is a parameter that accounts for the degeneracy of the level. For large values of the Gaussian broadening $\sigma_{i}$, it can be approximated by, $n_{\text {conf dot i }}$ $\approx N_{\text {dot }} g_{i}\left[1+\operatorname{erf}\left(F-E_{d i} / \sqrt{2} \sigma_{i}\right)\right]$, an approximation that suits better at lower temperatures and for strong broadening. Numerical integration is carried out in order to compute it in the general case.

Based on the following approximate relationship, 1/(1 $\left.+e^{x}\right) 1 /\left(1+e^{-x}\right) \approx 1 / \pi \sqrt{2 / \pi} e^{-2 x^{2} / \pi^{2}}$ and using Eqs. (A18)-(A20) in the Appendix, we developed the following analytical expression for the QD quasistatic contribution to the admittance:

$$
\lim _{\omega \rightarrow 0} C_{\mathrm{QD}}=\frac{\varepsilon S\left[1+\frac{q^{2}}{\varepsilon k T}\left(W-L_{\mathrm{conf}}\right) N_{\mathrm{dot}}\left(\frac{2}{\pi}\right)^{3 / 2} \times \sum_{i} g_{i} \frac{\sqrt{2} E_{t}}{\sqrt{\left(E_{t}^{2}+2 \sigma_{i}^{2}\right)}} e^{-\left(E_{F}-E_{d i}\right)^{2} /\left(E_{t}^{2}+\sigma_{i}^{2}\right)}\right]}{\left[W+L_{\mathrm{conf}} \frac{q^{2}}{\varepsilon k T}\left(W-L_{\mathrm{conf}}\right) N_{\mathrm{dot}}\left(\frac{2}{\pi}\right)^{3 / 2} \times \sum_{i} g_{i} \frac{\sqrt{2} E_{t}}{\sqrt{\left(E_{t}^{2}+2 \sigma_{i}^{2}\right)}} e^{-\left(E_{F}-E_{d i}\right)^{2} /\left(E_{t}^{2}+\sigma_{i}^{2}\right)}\right]},
$$

$$
\lim _{\omega \rightarrow 0} G_{\mathrm{QW}}=0 .
$$

With $n_{\text {conf }}=n_{\mathrm{QW}}+n_{\text {conf dot }}$ the quasistatic total admittance accounting for both the wetting layer and QDs confined systems is given by

$$
\lim _{\omega \rightarrow 0} C_{\mathrm{tot}}=\frac{\varepsilon S}{W} \frac{1+\frac{q^{2}}{\varepsilon k T}\left(W-L_{\mathrm{conf}}\right)\left[\frac{m_{c} k T}{\pi \hbar^{2}} \sum_{i} f_{0}\left(E_{w l 1}\right)+N_{\mathrm{dot}}\left(\frac{2}{\pi}\right)^{3 / 2} \times \sum_{i} g_{i} \frac{\sqrt{2} E_{t}}{\left.\sqrt{\left(E_{t}^{2}+2 \sigma_{i}^{2}\right)} e^{-\left(E_{F}-E_{d i}\right)^{2} /\left(E_{t}^{2}+\sigma_{i}^{2}\right)}\right]}\right.}{1+\frac{L_{\mathrm{conf}}}{W} \frac{q^{2}}{\varepsilon k T}\left(W-L_{\mathrm{conf}}\right)\left[\frac{m_{c} k T}{\pi \hbar^{2}} \sum_{i} f_{0}\left(E_{w l 1}\right)+N_{\mathrm{dot}}\left(\frac{2}{\pi}\right)^{3 / 2} \times \sum_{i} g_{i} \frac{\sqrt{2} E_{t}}{\sqrt{\left(E_{t}^{2}+2 \sigma_{i}^{2}\right)}} e^{-\left(E_{F}-E_{d i}\right)^{2} /\left(E_{t}^{2}+\sigma_{i}^{2}\right)}\right]},
$$

$\lim _{\omega \rightarrow 0} G_{\text {tot }}=0$.

\section{The delta-type layer with energy confinement lies outside the space charge region}

$$
W=\sqrt{\frac{2 \varepsilon\left(V+V_{b}\right)}{q N_{D}}} \text { and } \begin{aligned}
& \lim _{\omega \rightarrow 0} C_{\mathrm{tot}}=\frac{\varepsilon S}{W} \\
& \lim _{\omega \rightarrow 0} G_{\mathrm{tot}}=0
\end{aligned} .
$$

The quasistatic capacitance of the structure is determined by the homogeneous space charge region and is not affected by the presence of the delta-type thin layer with energy confinement. The charge in the delta-type layer is fully compensated by the charge in the depleted regions surrounding it (see equation (A2) in the Appendix). However, in principle, the contribution of the delta-type layer is measurable both in conduction and capacitance in the intermediate and high frequency regime (not in quasistatic conditions) and can also be used to provide information concerning the band discontinuities in heterostructures. ${ }^{12,13}$ 


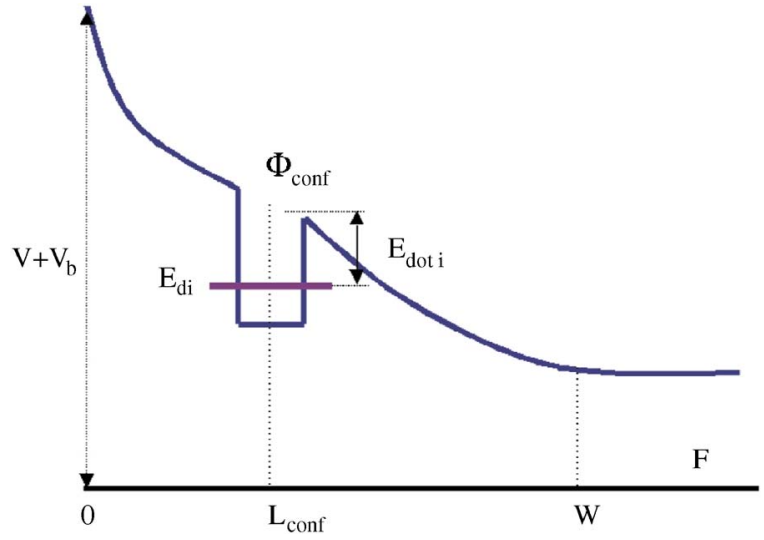

FIG. 6. (Color online) (Color onlie) Energy band diagram for the delta-type layer with energy confinement.

\section{Effects of a local field}

The Stark shift of an energy level in $p-i-n$ structures ${ }^{19-22}$ with a permanent electric dipole is given by ${ }^{16}$

$$
E=E_{0}+p F+\beta F^{2}
$$

where $p$ is the dipole (nm), $F$ is the electric field, and $\beta$ is the polarizability of the wave function in the dot (quantum confined Stark effect). The $\beta$ coefficient in a $\mathrm{QW}$ with infinite barriers is proportional to $L_{\mathrm{QW}}{ }^{4}$ In a first approximation for very thin layers, as is the case for our wetting layer and QDs, its contribution is negligible, i.e., the Stark shift in QDs is significantly smaller than in thick QWs.

Equation (13) can be rewritten as

$$
E \approx \beta\left(F-F_{0}\right)^{2}-\frac{p F_{0}}{2}
$$

where $F_{0}=p / 2 \beta$ is the built-in electric field for which the energy level $E$ has its maximum value. With no permanent dipole $F_{0}=0$ and there is a redshift when external bias is applied. If the field $F_{0}$ is of the order of $60 \mathrm{kV} / \mathrm{cm}$, the corresponding shift of an energy level $E$ due to the presence of a permanent dipole is $\Delta E=\sim-p F_{0} / 2 \sim 3 \mathrm{mV}$ for a $p$ value of $1 \mathrm{~nm}$ and we neglect it in our further analysis. Thus, in a first approximation we can use the model presented above to account for the presence of a local field by modifying the quantized energy levels with the quantity $V_{F} / 2$ such that $E_{\mathrm{dot} i}^{\prime}=E_{\mathrm{dot} i}+V_{F} / 2$ and the boundary condition at $z=0 \Phi=V+V_{b}$ becomes $\Phi=V+V_{b}+V_{F}$. For example, without the presence of a local field, when the region with energy confinement lies outside the space charge region $W$, the contribution of the electric field on each of its side changes its sign and the total field is zero, as evident from Fig. 5(a). In the presence of a local field, the potential and field profile shows an asymmetry of the field around $z=L_{\text {conf }}$ such that the sum is no longer zero, thus accounting for the local field. This field is related to the band bending $\Phi_{\text {conf }}$ by relationships similar to Eq. (A16) in the Appendix,

$$
\begin{aligned}
n_{\mathrm{conf}}= & N_{D}\left(w_{1}+w_{2}\right)=\sqrt{\frac{2 \varepsilon N_{d}\left(\Phi_{\mathrm{conf}}+\frac{V_{F}}{2}\right)}{q}} \\
& +\sqrt{\frac{2 \varepsilon N_{d}\left(\Phi_{\mathrm{conf}}-\frac{V_{F}}{2}\right)}{q}},
\end{aligned}
$$

and $\Phi_{\text {conf }}$ is obtained by solving the transcendental Eq. (15).

\section{Discussion}

In order to check the validity of the model for values of the parameters that are usually found in practice, Fig. 7 shows the comparison between the $C$ - $V$ profiles obtained using the analytical expression from Eq. (11) and the exact Poisson solution for $T=450 \mathrm{~K}$ (a) with and (c) without the contribution of the region near the QDs to the space charge region. Figure 7 also shows similar modeled dependencies for $T=50 \mathrm{~K}$, with (b) and without (d) the contribution of the region near the QDs to the space charge region, respectively. This contribution of the delta-type region with energy confinement to the space charge region is given by $w$ in Eq. (A16) from the Appendix and is meaningful only for the analytical solution, defining the applied bias for which the capacitance calculated using Eq. (12) $\left(W<L_{\text {conf }}\right)$ is replaced with the one calculated using Eq. (11) $\left(W>L_{\text {conf }}\right)$. We notice that the analytical expression, which is considerably faster than the exact solution of the Poisson equation, reproduces it accurately with the exception of few data points for which the space charge region reaches the QD layer. It can thus be used for finding the first rough values of the model parameters that fit the experimental data while the exact solution is used for refining them. From Figs. 7(a)-7(d), we find that the analytical solution has good accuracy for high temperatures $(300-450 \mathrm{~K})$ if the contribution $w$ is not taken into consideration and for low temperature $(50 \mathrm{~K})$ if it is taken into account, i.e., if the bias for which the expression in Eq. (11) starts to be applied is determined by the condition $W^{\prime}=W$ $+w>L_{\text {conf }}$ instead of $W>L_{\text {conf. }}$. The parameters used for modeling are as follows: $N_{D}=5.80 \times 10^{16} \mathrm{~cm}^{-3}, L_{\text {conf }}$ $=0.20 \mu \mathrm{m}, \quad V_{b}=1.340 \mathrm{~V}, V_{F}=0$ (no local field), $E_{1}$ $=0.100 \mathrm{eV} \quad$ (wetting layer), $E_{\text {dot } 1}=0.220 \mathrm{eV}, E_{\text {dot } 2}$ $=0.160 \mathrm{eV}, \quad E_{\text {dot } 3}=0.100 \mathrm{eV}, \sigma_{1}=\sigma_{2}=\sigma_{3}=0.060 \mathrm{eV}, g_{1}$ $=1, g_{2}=2, g_{3}=3$, and $N_{\mathrm{dot}}=3 \times 10^{10} \mathrm{~cm}^{-2}$.

After estimating the accuracy of the analytical solution, we check the consistency of our model by fitting more sets of experimental data for different temperatures: 30, 300, and $450 \mathrm{~K}$. The results are plotted in Figs. 4(a) and 4(b). As explained above, the kink in the $C-V$ plots defined by the bias for which the bulk space charge region reaches the delta-type thin film with energy confinement (wetting layer and QDs) can only be well reproduced using the exact Poisson solution. Even so, there is a consistent discrepancy for all temperatures between the modeled results and the experimental data in this region. The fit can be made acceptable if the presence of a local field in the QDs is taken into consideration, as mentioned in Ref. 5. The value of the local field, which accounts for the charge separation resulting from local 

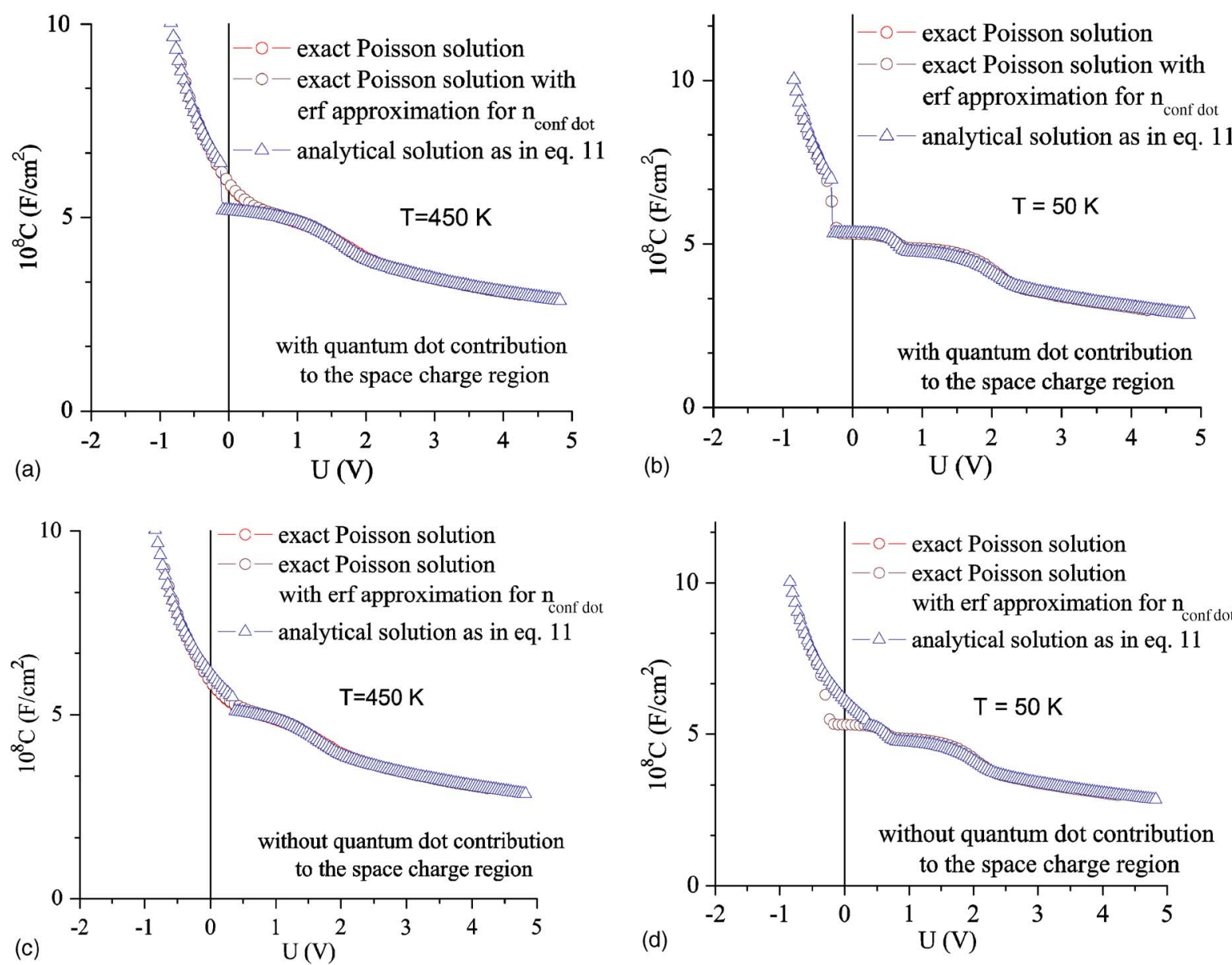

FIG. 7. (Color online) Comparison between the $C$ - $V$ profiles obtained using the analytical expression from Eq. (14) and the exact Poisson solution for $T$ $=450 \mathrm{~K}$ (a) with and (c) without the contribution of the region near the QD to the space charge region and $T=50 \mathrm{~K}$ (b) with and (d) without the contribution of the region near the QD to the space charge region, respectively. The parameters used for modeling are as follows: $N d=5.80 \times 10^{16} \mathrm{~cm}^{-3}, L \mathrm{conf}$ $=0.20 \mu \mathrm{m}, V_{b}=1.340 \mathrm{~V}, V_{F}=0$ (no local field) $E_{1}=0.100 \mathrm{eV}$ (wetting layer), $E_{\mathrm{dot} 1}=0.220 \mathrm{eV}, E_{\mathrm{dot} 2}=0.160 \mathrm{eV}, E_{\mathrm{dot} 3}=0.100 \mathrm{eV} ; \sigma_{1}=0.060 \mathrm{eV}, \sigma_{2}$ $=0.060 \mathrm{eV}, \sigma_{3}=0.060 \mathrm{eV}, g_{1}=1, g_{2}=2, g_{3}=3$, and $N_{\mathrm{dot}}=3 \times 10^{10} \mathrm{~cm}^{-2}$.

strain and composition variations in Ref. 4, is $25 \mathrm{kV} / \mathrm{cm}$, but its orientation was not mentioned. For our case, the value of the local field that gives the best fit in the plots in Fig. 4 corresponds to a value of $V_{F}$ of $50 \mathrm{mV}$, which corresponds to a value of the local field of $28 \mathrm{kV} / \mathrm{cm}$ for a dot height of 1.8 $\mathrm{nm}$ estimated from transmission electron microscopy images (not shown here), similar to the one reported in Ref. 4.

The growth method is not mentioned in Ref. 4. To our knowledge, the available values of the local field reported in literature are for QDs grown by molecular beam epitaxy (MBE), while the QDs in the present studies were grown by MOCVD. The growth temperatures for the MOCVD method are typically $50-100{ }^{\circ} \mathrm{C}$ higher than those for MBE and the growth parameters can have a crucial influence on the optolectronic device parameters such as threshold current and emission wavelength in laser diodes for example. It was shown in Refs. 19 and 20 that the internal dipole field in MOCVD grown dots can have large values of the order of $150 \mathrm{kV} / \mathrm{cm}$, while in the MBE grown material it is much smaller. The presence of a large internal electric field can reduce the efficiency of the QD PL emission due to the reduced overlap of the electron and hole wave functions. However, the internal field reported in Ref. 19 is oriented from the base of the dot toward its apex, while in our case its orientation is from the apex to the base, i.e., inverted electron-hole alignment meaning that is contrary to the theoretically predicted orientation for a dot with a uniform In composition and that it can only be explained by a composition gradient within the dot. A similar orientation was reported for MBE grown dots, even though with a significantly lower amplitude of the local field. ${ }^{23}$ The orientation of the field can strongly depend on the specific growth parameters, however. In our case, we also experimentally observe a significantly smaller blueshift than that reported in Ref. 20 between the electroluminescence and photocurrent peaks (not shown here) and the low intensity PL peaks, consistent with a different orientation of the dipole.

Another important issue resulting from modeling is the conduction band energy structure. The $C$ - $V$ plots in Figs. 4(a) and 4(b) can be fitted with both sets of parameters:
(a) $\quad E_{\text {dot } 1}=0.180 \mathrm{eV}, \quad \sigma_{1}=180 \mathrm{meV}, \quad g_{1}=3, \quad E_{\text {dot } 2}$ $=0.375 \mathrm{eV}, \quad \sigma_{2}=200 \mathrm{meV}, \quad g_{2}=4, \quad$ and $E_{w l} 1$ $=0.090 \mathrm{eV}$ and
(b) $\quad E_{\text {dot } 1}=0.180 \mathrm{eV}, \quad \sigma_{1}=180 \mathrm{meV}, \quad g_{1}=3, \quad E_{\text {dot } 2}$ $=0.375 \mathrm{eV}, \quad \sigma_{2}=200 \mathrm{meV}, \quad g_{2}=4$ and $E_{\text {dot } 3}$ $=0.090 \mathrm{eV}, \sigma_{3}=80 \mathrm{meV}, g_{3}=1$.


The issue here is in the assignment of the level found 90 meV below the conduction band of the bulk, i.e., whether it would be assigned to a wetting layer or QD state. The PL spectra suggest that it is a wetting layer state. If we take the conduction band discontinuity between $\operatorname{In}_{0.50} \mathrm{Ga}_{0.50} \mathrm{As}$ and GaAs to be $265 \mathrm{meV}$ (Ref. 4) and the following relationship between the confining energy of a very thin delta-type wetting layer and the band offset: ${ }^{24} E_{1}=m_{c} L_{\mathrm{QW}}^{2} \Delta E_{c}^{2} / 2 \hbar^{2}$ and using $E_{1}=90 \mathrm{meV}$, we extract the thickness of the wetting layer as $L_{\mathrm{QW}}=1.7 \mathrm{~nm}$, close to 5-6 ML. It is thus reasonable to assign it to a wetting layer state rather than to a QD state. It is worth mentioning, however, that to our knowledge there are no previous reports concerning the observation of wetting layer states in $C$ - $V$ plots together with their parameters extracted from the exact modeling. Energy confinements around 170 and $90 \mathrm{meV}$ are typically found in MBE grown dots and are usually assigned to the ground and excited dot states, ${ }^{4,7}$ even though in some cases the band structure deduced from $C-V$ measurements does not match the one from PL. ${ }^{4}$ The difference between the ground and excited states typically extracted from $C-V$ modeling is $80 \mathrm{meV}$, while the energy splitting in PL spectra is around $45 \mathrm{meV}{ }^{4}$ The difference was attributed to the Coulomb effects, which can account for $20 \mathrm{meV}$ energy when charging an additional electron in a typical disk shaped dot with radius of $10 \mathrm{~nm}^{4}$ However, this effect should depend on the degeneracy factors of the dot levels, which are very difficult to estimate and does not influence the wetting layer states. In our case, we definitely have a much stronger contribution from the wetting layer state emission than in previously reported QDs and these states are also expected to influence the $C$ - $V$ profiles. The degeneracy of excited dot state tends to increase for higher energy levels while in our case if we attribute the level with $90 \mathrm{meV}$ confinement to QDs its degeneracy factor deduced from modeling would be $g=1$, compared to $g=3$ for the lower level, which is very unlikely.

We thus attribute the state with $90 \mathrm{meV}$ confinement to the wetting layer and the state with $180 \mathrm{meV}$ confinement to QD states [see Fig. 8(a)] for the diagram showing the corresponding energy levels. Assuming a $60 \%$ value of the ratio $\Delta E_{c} / \Delta E_{g}$, the PL transition of $1.130 \mathrm{eV}$ would correspond to a confinement energy of $175 \mathrm{meV}$ in the conduction band with respect to the GaAs bulk, in good agreement with the value of $180 \mathrm{meV}$ deduced from $C$ - $V$ fitting of the experimental data. The lower PL transition would correspond to an energy confinement of $220 \mathrm{meV}$ but the transition broadening due to size fluctuations is expected to be larger for conduction band states than for interband transitions because of smaller effective mass of electrons, and thus it is not resolved. Instead, from $C-V$ modeling we extract a broad level $(180 \mathrm{meV})$ around the confinement energy of $180 \mathrm{meV}$ below the GaAs conduction band edge. The total broadening of the PL interband transitions would thus be 2.5 times less than for the conduction band state deduced from $C$ - $V$ modeling, as shown in Fig. 8(b).

From $C$ - $V$ fitting we also extract a level with $375 \mathrm{meV}$ confinement, below the value of the conduction band discontinuity of $265 \mathrm{meV}$. This obviously cannot be attributed to a

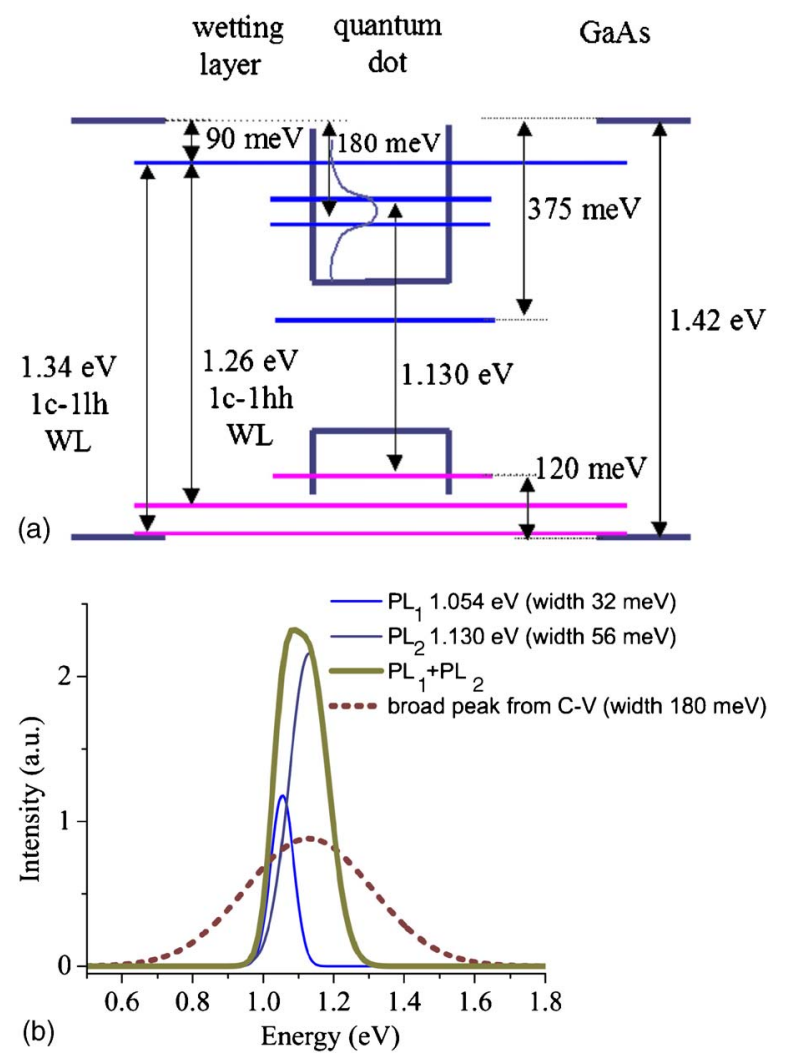

FIG. 8. (Color online) (a) Diagram of the band structure showing the wetting layer and QD transitions and (b) deconvolution of PL dot interband transitions compared with the state derived from $C-V$ fitting of the experimental data.

quantized level in the QD or wetting layer and is not reported previously for MBE grown dots. We attribute it to a defect level that contributes to the low PL efficiency of the dot states in our structures, in addition to the low wave function overlap due to the local field. In the presence of defects, the characteristic step in the quasistatic $C$ - $V$ profile can still be observed, ${ }^{25}$ even though the thermal activation of the perpendicular conductivity of the structure may be governed by the deep levels rather than by thermionic emission from quantized levels. ${ }^{25}$ A defect level might also explain why we do not observe an evident step in the capacitance versus frequency behavior (not shown) both at $450 \mathrm{~K}$ and at low temperatures in the range of $1 \mathrm{~Hz}-100 \mathrm{kHz}$. A similar behavior was reported recently in Ref. 26 and it was pointed out that in some structures the typical frequency dispersion in admittance spectra is observed and in others with similar dots it is not, depending on the specific growth parameters, even though the exact cause of this behavior is not clear.

\section{CONCLUSIONS}

This paper derives an analytical expression for the quasistatic capacitance of a QD layer embedded in a junction, where the reverse bias is used to discharge the initially occupied energy levels. This analysis can be used to determine the position and the Gaussian homogeneous broadening of the energy levels in the conduction band and is applied for an InGaAs/GaAs QD structure grown by MOCVD. It is shown that the Gaussian broadening of the conduction band levels is 
significantly larger than the broadening of the interband PL transitions involving both conduction and hole states. The analysis also reveals a contribution from the wetting layer rather than from an excited dot transition in the quasistatic $C-V$ profile. The presence of a built-in local field whose orientation is from the apex of the dot toward its base, contrary to the direction expected for a strained dot with uniform composition (negative dipole), is also derived from the fit of the experimental $C-V$ data.

\section{ACKNOWLEDGMENTS}

This work was financially supported by the Romanian Ministry of Education and Research in the frame of the nucleus project 41N/2006. Australian authors gratefully acknowledge financial support from the Australian Research Council. M. Buda also gratefully acknowledges the financial support from the Australian Research Council for her visit at ANU, Australia.

\section{APPENDIX: CURRENT MODEL FOR CAPACITANCE OF A QW THIN FILM WITH ENERGY CONFINEMENT EMBEDDED IN A LAYER WITH UNIFORM COMPOSITION}

The delta-type thin layer with energy confinement lies inside the space charge region

By integrating the Poisson equation, we obtain:

$$
\begin{aligned}
-\left(V+V_{b}\right)= & q \frac{N_{D} W^{2}}{2 \varepsilon}-q \frac{n_{\mathrm{conf}} L_{\mathrm{conf}}}{\varepsilon} \rightarrow \frac{\partial W}{\partial V} \\
& =\frac{-1+\frac{q L_{\mathrm{conf}}}{\varepsilon} \frac{\partial n_{\mathrm{conf}}}{\partial V}}{W \frac{q N_{D}}{\varepsilon}} .
\end{aligned}
$$

The total charge in the structure is given by

$$
Q=S q\left(N_{D} W-n_{\text {conf }}\right) \text {. }
$$

We then obtain

$$
\begin{aligned}
\frac{\partial Q}{\partial V} & =\frac{S q\left(N_{D} d W-d n_{\mathrm{conf}}\right)}{\left(-\frac{q N_{D} W}{\varepsilon} d W+\frac{q L_{\mathrm{conf}}}{\varepsilon} d n_{\mathrm{conf}}\right)} \\
& =-\frac{S q\left(N_{D} \frac{\partial W}{\partial V}-\frac{\partial n_{\mathrm{conf}}}{\partial V}\right)}{\left(-\frac{q N_{D} W}{\varepsilon} \frac{\partial W}{\partial V}+\frac{q L_{\mathrm{conf}}}{\varepsilon} \frac{\partial n_{\mathrm{conf}}}{\partial V}\right)},
\end{aligned}
$$

and thus by substituting $\partial W / \partial V$ from Eq. (A1) into Eq. (A3), we have

$$
\begin{aligned}
& C=\varepsilon \frac{S}{W}+q S\left(1-\frac{L_{\mathrm{conf}}}{W}\right) \times \operatorname{real}\left(\frac{\partial n_{\mathrm{conf}}}{\partial V}\right), \\
& G=-q S \nu\left(1-\frac{L_{\mathrm{conf}}}{W}\right) \times \operatorname{imag}\left(\frac{\partial n_{\mathrm{conf}}}{\partial V}\right) .
\end{aligned}
$$

We then look for the relationship between $\partial n_{\text {conf }} / \partial V$ and the band structure of the region with energy confinement. From Eq. (A2) and using Eq. (A5) and (A6), we obtain $\partial n_{\text {conf }} / \partial \Phi_{\text {conf }}$ in Eq. (A7)

$$
\begin{aligned}
& -\left(V+V_{b}\right)=q \frac{N_{D} W^{2}}{2 \varepsilon}-q \frac{n_{\mathrm{conf}} L_{\mathrm{conf}}}{\varepsilon}, \\
& \Phi_{\mathrm{conf}}=-\frac{q N_{D}}{2 \varepsilon}\left(W-L_{\mathrm{conf}}\right)^{2}, \\
& \frac{\partial \Phi_{\mathrm{conf}}}{\partial V}=\left(1-\frac{L_{\mathrm{conf}}}{W}\right)\left(1-\frac{q L_{\mathrm{conf}}}{\varepsilon} \frac{\partial n_{\mathrm{conf}}}{\partial V}\right) .
\end{aligned}
$$

It follows that

$$
\frac{\partial n_{\mathrm{conf}}}{\partial \Phi_{\mathrm{conf}}}=\frac{\partial n_{\mathrm{conf}}}{\partial V} \frac{\partial V}{\partial \Phi_{\mathrm{conf}}} .
$$

Following Ref. 18, we have the following relationship for the small changes in the occupancy factor $\partial f(E, t)$ of the state with energy $E_{i}$ at time $t$, describing the kinetics of the carrier capture and escape processes:

$$
\partial f[(E, t)]=\frac{f_{0}(E)\left[1-f_{0}(E)\right]}{1+j \omega \frac{f_{0}(E)}{c_{n} n_{s 0}}} \frac{\partial n_{s}(t)}{n_{s 0}}, \quad n_{s 0}=N_{D} e^{\Phi_{\text {conf }} / E_{t}}
$$

where $n_{s 0}$ is the free carrier concentration at equilibrium in the conduction band of the bulk material, $c_{n}$ is the capture emission coefficient of these carriers by the quantized level $E_{i}$, and the steady state occupancy $f_{0}$ is given by the FermiDirac function. From Eq. (A8), it follows that

$$
\partial f(E, t)=\frac{f_{0}(E)\left[1-f_{0}(E)\right]}{1+j \omega \frac{f_{0}(E)}{c_{n} n_{s 0}}} \frac{\partial \Phi_{\mathrm{conf}}}{E_{t}} .
$$

We then have

$$
\partial n_{\mathrm{conf}}(t)=\frac{\partial \Phi_{\mathrm{conf}}}{E_{t}} \sum_{i} \int_{E_{i}}^{\infty} D_{i} \times \frac{f_{0}\left(1-f_{0}\right)}{1+j \omega \frac{f_{0}}{c_{n} n_{s 0}}} d E
$$

where $D_{i}$ is the density of states and using Eq. (A7), we obtain

$$
\begin{aligned}
\frac{\partial n_{\mathrm{conf}}}{\partial \Phi_{\mathrm{conf}}} & =\frac{\frac{\partial n_{\mathrm{conf}}}{\partial V}}{\left(1-\frac{L_{\mathrm{conf}}}{W}\right)\left(1-\frac{q L_{\mathrm{conf}}}{\varepsilon} \frac{\partial n_{\mathrm{conf}}}{\partial V}\right)} \\
& =\frac{1}{E_{t}} \sum_{i} \int_{E_{i}}^{\infty} D_{i} \times \frac{f_{0}\left(1-f_{0}\right)}{1+j \omega \frac{f_{0}}{c_{n} n_{s 0}}} d E .
\end{aligned}
$$




$$
\frac{\partial n_{\mathrm{QW}}}{\partial \Phi_{\mathrm{QW}}}=\frac{1}{E_{t}} \sum_{i} \int_{E_{i}}^{\infty} \frac{m}{\pi \hbar^{2}} \times \frac{f_{0}\left(1-f_{0}\right)}{1+j \omega \frac{f_{0}}{c_{n} n_{s 0}}} d E=\sum_{i}\left(c_{i}-j d_{i}\right)
$$

and thus

$$
\begin{aligned}
& \frac{\partial n_{\mathrm{QW}}}{\partial V}=\frac{\sum_{i}\left(c_{i}-j d_{i}\right)}{1+\frac{q L_{\mathrm{conf}}}{\varepsilon} \times \sum_{i}\left(c_{i}-j d_{i}\right)} \\
& c_{i}=\frac{q D_{i}}{\omega \tau_{i}}\left(1-\frac{L_{\mathrm{conf}}}{W}\right) \times \operatorname{arctg}\left[\omega \tau_{i} f_{0}\left(E_{\mathrm{wl} i}\right)\right] \\
& d_{i}=\frac{q D_{i}}{2 \omega \tau_{i}}\left(1-\frac{L_{\mathrm{conf}}}{W}\right) \times \ln \left[1+\left(\omega \tau_{i} f_{0}\left(E_{\mathrm{wl} i}\right)\right)^{2}\right]
\end{aligned}
$$

are obtained by integrating Eq. (A12) with $\tau_{i}=1 / c_{n i}\left(E_{i}\right) n_{s 0}$. Under steady state conditions, we then obtain

$$
\begin{aligned}
\lim _{\omega \tau_{i} \rightarrow 0} c_{i} & =\lim _{\omega \tau_{i} \rightarrow 0}\left\{\frac{q D_{i}}{\omega \tau_{i}}\left(1-\frac{L_{\mathrm{conf}}}{W}\right) \times \operatorname{arctg}\left[\omega \tau_{i} f_{0}\left(E_{\mathrm{wl} i}\right)\right]\right\} \\
& =\mathrm{q} D_{i}\left(1-\frac{\mathrm{L}_{\mathrm{conf}}}{W}\right) \times \mathrm{f}_{0}\left(E_{\mathrm{wl} i}\right),
\end{aligned}
$$

$$
\lim _{\omega \tau_{i} \rightarrow 0} d_{i}=0,
$$

and thus

$$
\begin{aligned}
\lim _{\omega \tau_{i} \rightarrow 0}\left(\frac{\partial n_{\mathrm{QW}}}{\partial V}\right) \\
=\frac{\left(1-\frac{L_{\mathrm{conf}}}{W}\right) \frac{q m_{c}}{\pi \hbar^{2}} \times \sum_{i} f_{0}\left(E_{\mathrm{wl} i}\right)}{1+\frac{q L_{\mathrm{conf}}}{\varepsilon}\left(1-\frac{L_{\mathrm{conf}}}{W}\right) \frac{q m_{c}}{\pi \hbar^{2}} \times \sum_{i} f_{0}\left(E_{\mathrm{wl} i}\right)} .
\end{aligned}
$$

\section{The delta-type thin layer with energy confinement lies outside the space charge region}

In this case, the charge in the delta-type thin region with energy confinement is compensated by carriers from the bulk depleted regions surrounding it, and thus the total charge $Q$ outside the space charge region $W$ is zero. We thus have

$$
\begin{aligned}
& \mathrm{n}_{\mathrm{conf}}=2 w N_{D}, \\
& \Phi_{\mathrm{QW}}=\frac{q N_{D}}{2 \varepsilon} w^{2},
\end{aligned}
$$

where $w$ is the thickness of the space charge region at the sides of the quantum confined region. It follows that we can obtain the value of the potential $\Phi_{\text {conf }}$ by solving the transcendental Eq. (A17),

$$
n_{\mathrm{conf}}=\sqrt{\frac{8 N_{D} \varepsilon \Phi_{\mathrm{QW}}}{q}},
$$

where $n_{\text {conf }}$ is given by Eq. (3) in the main paper.

Under quasistatic conditions, the capacitance is not affected by the presence of the delta-type thin layer with energy confinement and we have

$C=\varepsilon S / W$, where $W$ is the thickness of the space charge region in the bulk.

\section{Derivation of the analytical expression for the QD contribution to capacitance}

For the QDs, similar to Eqs. (A12) and (A13) for a QW, we have

$$
\frac{\partial n_{\mathrm{conf} \text { dot }}}{\partial V}=\frac{\frac{q}{k T}\left(1-\frac{L_{\mathrm{conf}}}{W}\right) \times \sum_{i}\left(a_{i}+j b_{i}\right)}{\left[1+\frac{q L}{\varepsilon} \frac{q^{2}}{k T}\left(1-\frac{L_{\mathrm{conf}}}{W}\right) \times \sum_{i}\left(a_{i}+j b_{i}\right)\right]}
$$

with the coefficients $a_{i}$ and $b_{i}$ given by $a_{i}+j b_{i}=I_{i, \mathrm{QD}}$,

$$
\begin{aligned}
I_{i, \mathrm{QD}} & =\frac{2 N_{\mathrm{dot}} g_{i}}{\sqrt{2 \pi} \sigma_{i}} \int_{-\infty}^{\infty} e^{-\left(E-E_{d i}\right)^{2} / 2 \sigma_{i}^{2}} \times \frac{f_{0}\left(1-f_{0}\right)}{\left(1+j \frac{\omega f_{0}}{c_{n} n_{s 0}}\right)} d E \\
& =-\frac{2 N_{\mathrm{dot}} g_{i}}{\sqrt{2 \pi} \sigma_{i}} E_{t} \int_{-\infty}^{\infty} e^{-\left(E-E_{d i}\right)^{2} / 2 \sigma_{i}^{2}} \times \frac{d f_{0}}{\left(1+j \frac{\omega f_{0}}{c_{n} n_{s 0}}\right)},
\end{aligned}
$$

and thus

$$
\begin{aligned}
I_{i, \mathrm{QD}}= & -\frac{2 N_{\mathrm{dot} g} g_{i}}{\sqrt{2 \pi} \sigma_{i}} E_{t} \int_{-\infty}^{\infty} e^{-\left(E-E_{d i}\right)^{2} / 2 \sigma_{i}^{2}} \\
& \times \frac{\left(1-j \frac{\omega f_{0}}{c_{n} n_{s 0}}\right)}{\left[1+\left(\frac{\omega f_{0}}{c_{n} n_{s 0}}\right)^{2}\right]} d f_{0} .
\end{aligned}
$$

For quasistatic conditions and taking into account that $1 /\left(1+e^{x}\right) 1 /\left(1+e^{-x}\right) \approx 1 / \pi \sqrt{2 / \pi}\left(e^{-2 x^{2} / \pi^{2}}\right)$, we obtain

$$
\begin{aligned}
\lim _{\omega \rightarrow 0} I_{i, \mathrm{QD}}= & \frac{2 N_{\mathrm{dot}} g_{i}}{\sqrt{2 \pi} \sigma_{i}} \frac{1}{\pi} \sqrt{\frac{2}{\pi}} \int_{-\infty}^{+\infty} \\
& \times e^{\left[-\left(E-E_{d i}\right)^{2} / 2 \sigma_{i}^{2}-\left(E-E_{F}\right)^{2} / E_{t}^{2}\right]} d E+j 0,
\end{aligned}
$$


and thus

$$
\begin{aligned}
\lim _{\omega \rightarrow 0} I_{i, \mathrm{QD}}= & \left(\frac{2}{\pi}\right)^{3 / 2} \frac{N_{\mathrm{dot}} g_{i}}{\sigma_{i}} \frac{1}{\sqrt{\frac{1}{2 \sigma_{i}^{2}}+\frac{1}{E_{t}^{2}}}} \\
& \times e^{-\left(E_{F}-E_{d i}\right)^{2} /\left(E_{t}^{2}+2 \sigma_{i}^{2}\right)}+j 0 .
\end{aligned}
$$

The QD contribution to the quasistatic capacitance is given by

$$
\lim _{\omega \rightarrow 0} C_{\mathrm{QD}}=\frac{\varepsilon S\left[1+\frac{q^{2}}{\varepsilon k T}\left(W-L_{\mathrm{conf}}\right) \times \sum_{i} \lim _{\nu \rightarrow 0} I_{i, \mathrm{QD}}\right]}{\left[W+L_{\mathrm{conf}} \frac{q^{2}}{\varepsilon k T}\left(W-L_{\mathrm{conf}}\right) \times \sum_{i} \lim _{\nu \rightarrow 0} I_{i, \mathrm{QD}}\right]},
$$

$$
\lim _{\omega \rightarrow 0} G_{\mathrm{QD}}=0,
$$

and thus the final expression for the capacitance of a structure with a delta-type QD layer embedded at $z=L_{\text {conf }}$ is given by

$$
\lim _{\omega \rightarrow 0} C_{\mathrm{QD}}=\frac{\varepsilon S\left[1+\frac{q^{2}}{\varepsilon k T}\left(W-L_{\mathrm{conf}}\right) N_{\mathrm{dot}}\left(\frac{2}{\pi}\right)^{3 / 2} \times \sum_{i} g_{i} \frac{\sqrt{2} E_{t}}{\sqrt{\left(E_{t}^{2}+2 \sigma_{i}^{2}\right)}} e^{-\left(E_{F}-E_{d i}\right)^{2} /\left(E_{t}^{2}+\sigma_{i}^{2}\right)}\right]}{\left[W+L_{\mathrm{conf}} \frac{q^{2}}{\varepsilon k T}\left(W-L_{\mathrm{conf}}\right) N_{\mathrm{dot}}\left(\frac{2}{\pi}\right)^{3 / 2} \times \sum_{i} g_{i} \frac{\sqrt{2} E_{t}}{\sqrt{\left(E_{t}^{2}+2 \sigma_{i}^{2}\right)}} e^{-\left(E_{F}-E_{d i}\right)^{2} /\left(E_{t}^{2}+\sigma_{i}^{2}\right)}\right]},
$$

$\lim _{\omega \rightarrow 0} G_{\mathrm{QD}}=0$.

${ }^{1}$ D. Granados and J. M. Garcia, Nanotechnology 16, S282 (2005).

${ }^{2}$ P. N. Brunkov, A. Patane, A. Levin, L. Eaves, and P. C. Main, Phys. Rev. B 65, 085326 (2002).

${ }^{3}$ W.-H. Chang, W. Y. Chen, M. C. Cheng, C. Y. Lai, T. M. Hsu, N.-T. Yeh, and J.-I. Chyi, Phys. Rev. B 64, 125315 (2001).

${ }^{4}$ W.-H. Chang and T. M. Hsu, Phys. Rev. B 62, 13040 (2000).

${ }^{5}$ R. Wetzler, A. Wacker, and E. Scholl, Appl. Phys. Lett. 77, 1671 (2000).

${ }^{6}$ P. N. Brunkov, A. R. Kovsh, V. M. Ustinov, Yu. G. Musikhin, N. N. Ledentsov, S. G. Konnikov, A. Polimeni, A. Patane, P. C. Main, L. Eaves, and C. M. A. Kapteyn, J. Electron. Mater. 28, 486 (1999).

${ }^{7}$ P. N. Brounkov, A. Polimeni, S. T. Stoddart, M. Henini, L. Eaves, P. C. Main, A. R. Kovsh, Yu. G. Musikhin, and S. G. Konnikov, Appl. Phys. Lett. 73, 1092 (1998).

${ }^{8}$ G. Medeiros-Ribeiro, D. Leonard, and P. M. Petroff, Appl. Phys. Lett. 66, 1767 (1995).

${ }^{9}$ V. I. Zubkov, M. A. Melnik, A. V. Solomonov, E. O. Tselev, F. Bugge, M. Weyers, and G. Trankle, Phys. Rev. B 70, 075312 (2004).

${ }^{10}$ C. R. Moon, B.-D. Choe, S. D. Kwon, H. K. Shin, and H. Lim, J. Appl. Phys. 84, 2673 (1998).

${ }^{11}$ P. N. Brounkov, T. Bennyatou, and G. Guillot, J. Appl. Phys. 80, 864 (1996).

${ }^{12}$ Y. B. Jia, H. G. Grimmeiss, Z. Y. Han, and L. Dobaczewski, Semicond. Sci. Technol. 11, 1672 (1996).

${ }^{13}$ L. F. Marsal, J. M. Lopez-Villegas, J. Bosh, and J. R. Morante, J. Appl. Phys. 76, 1077 (1994).

${ }^{14}$ X. Letartre, D. Stievenard, and M. Lannoo, J. Appl. Phys. 68, 116 (1990).

${ }^{15}$ C. M. A. Kapteyn, F. Heinrichsdorff, O. Stier, R. Heitz, M. Grundmann, N. D. Zakharov, and D. Bimberg, Phys. Rev. B 60, 14265 (1999).
${ }^{16}$ G. Medeiros-Ribeiro, J. M. Garcia, and P. M. Petroff, Phys. Rev. B 56, 3609 (1997).

${ }^{17}$ H. H. Tan, K. Sears, S. Mokkapati, L. Fu, Y. Kim, P. McGowan, M. Buda, and C. Jagadish, IEEE J. Sel. Top. Quantum Electron. 12, 1242 (2006).

${ }^{18}$ J. M. Lopez-Villegas, P. Roura, J. Bosch, and J. R. Morante, J. Electrochem. Soc. 140, 1492 (1993).

${ }^{19}$ A. Passaseo, G. Maruccio, M. de Vittorio, S. de Rinaldis, T. Todaro, R. Rinaldi, and R. Cingolani, Appl. Phys. Lett. 79, 1435 (2001).

${ }^{20}$ A. Passaseo, M. de Vittorio, M. T. Todaro, I. Tarantini, M. de Giorgi, R. Cingolani, A. Taurino, M. Catalano, A. Fiore, A. Markus, J. X. Chen, C. Paranthoen, U. Oesterle, and M. Ilegems, Appl. Phys. Lett. 82, 3632 (2003).

${ }^{21}$ W. Sheng and J.-P. Leburton, Phys. Rev. B 67, 125308 (2003).

${ }^{22}$ P. W. Fry, I. E. Itskevich, D. J. Mowbray, M. S. Skolnick, J. J. Finley, J. A. Barker, E. P. O'Reilly, L. R. Wilson, I. A. Larkin, P. A. Maksym, M. Hopkinson, M. Al-Khafaji, J. P. R. David, A. G. Cullis, G. Hill, and J. C. Clark, Phys. Rev. Lett. 84, 733 (2000).

${ }^{23}$ P. W. Fry, I. E. Itskevich, D. J. Mowbray, M. S. Skolnick, J. J. Finley, J. A. Barker, E. P. O'Reilly, L. R. Wilson, I. A. Larkin, P. A. Maksym, M. Hopkinson, M. Al-Khafaji, J. P. R. David, A. G. Cullis, G. Hill, and J. C. Clark, Phys. Rev. Lett. 84, 733 (1999).

${ }^{24}$ J. Brubach, A. Yu. Silov, J. E. M. Haverkort, W. van der Vleuten, and J. H. Wolter, Superlattices Microstruct. 21, 527 (1997).

${ }^{25}$ P. N. Brounkov, T. Benyattou, G. Guillot, and S. A. Clark, J. Appl. Phys. 77, 240 (1995).

${ }^{26}$ E. Gombia, R. Mosca, S. Franchi, P. Frigeri, and C. Ghezzi, Mater. Sci. Eng., C C26, 867 (2006). 